\title{
Little evidence for enhanced phenotypic evolution in early teleosts relative to their living fossil sister group
}

\author{
John T. Clarke ${ }^{a, b, 1}$, Graeme T. Lloyd c', and Matt Friedman ${ }^{b, 2}$ \\ ${ }^{a}$ Department of Earth and Environmental Science, University of Pennsylvania, Philadelphia, PA 19104-6316; ${ }^{\circ}$ Department of Earth Sciences, University \\ of Oxford, Oxford OX1 3AN, United Kingdom; and 'Department of Biological Sciences, Faculty of Science, Macquarie University, North Ryde, NSW
} 2109, Australia

Edited by Neil H. Shubin, University of Chicago, Chicago, IL, and approved August 19, 2016 (received for review May 6, 2016)

Since Darwin, biologists have been struck by the extraordinary diversity of teleost fishes, particularly in contrast to their closest "living fossil" holostean relatives. Hypothesized drivers of teleost success include innovations in jaw mechanics, reproductive biology and, particularly at present, genomic architecture, yet all scenarios presuppose enhanced phenotypic diversification in teleosts. We test this key assumption by quantifying evolutionary rate and capacity for innovation in size and shape for the first 160 million y (Permian-Early Cretaceous) of evolution in neopterygian fishes (the more extensive clade containing teleosts and holosteans). We find that early teleosts do not show enhanced phenotypic evolution relative to holosteans. Instead, holostean rates and innovation often match or can even exceed those of stem-, crown-, and total-group teleosts, belying the living fossil reputation of their extant representatives. In addition, we find some evidence for heterogeneity within the teleost lineage. Although stem teleosts excel at discovering new body shapes, early crown-group taxa commonly display higher rates of shape evolution. However, the latter reflects low rates of shape evolution in stem teleosts relative to all other neopterygian taxa, rather than an exceptional feature of early crown teleosts. These results complement those emerging from studies of both extant teleosts as a whole and their sublineages, which generally fail to detect an association between genome duplication and significant shifts in rates of lineage diversification.

neopterygian | phylogeny | genome duplication | fossil record | morphological diversification

$\mathbf{N}$ umbering 29,000 species, teleost fishes account for half of modern vertebrate richness. In contrast, their holostean sister group, consisting of gars and the bowfin, represents a mere eight species restricted to the freshwaters of eastern North America (1). This stark contrast between teleosts and Darwin's original "living fossils" (2) provides the basis for assertions of teleost evolutionary superiority that are central to textbook scenarios $(3,4)$. Classic explanations for teleost success include key innovations in feeding $(3,5)$ (e.g., protrusible jaws and pharyngeal jaws) and reproduction $(6,7)$. More recent work implicates the duplicate genomes of teleosts (8-10) as the driver of their prolific phenotypic diversification $(8,11-13)$, concordant with the more general hypothesis that increased morphological complexity and innovation is an expected consequence of genome duplication $(14,15)$.

Most arguments for enhanced phenotypic evolution in teleosts have been asserted rather than demonstrated $(8,11,12,15,16$; but see ref. 17), and draw heavily on the snapshot of taxonomic and phenotypic imbalance apparent between living holosteans and teleosts. The fossil record challenges this neontological narrative by revealing the remarkable taxonomic richness and morphological diversity of extinct holosteans (Fig. 1) $(18,19)$ and highlights geological intervals when holostean taxonomic richness exceeded that of teleosts (20). This paleontological view has an extensive pedigree. Darwin (2) invoked a long interval of cryptic teleost evolution preceding the late Mesozoic diversification of the modern radiation, a view subsequently supported by the implicit (18) or explicit (19) association of Triassic-Jurassic species previously recognized as "holostean ganoids" with the base of teleost phylogeny. This perspective became enshrined in mid-20th century treatments of actinopterygian evolution, which recognized an early-mid Mesozoic phase dominated by holosteans sensu lato and a later interval, extending to the modern day, dominated by teleosts $(4,20,21)$. Contemporary paleontological accounts echo the classic interpretation of modest teleost origins (22-24), despite a systematic framework that substantially revises the classifications upon which older scenarios were based (22-25). Identification of explosive lineage diversification in nested teleost subclades like otophysans and percomorphs, rather than across the group as a whole, provides some circumstantial neontological support for this narrative (26).

In contrast to quantified taxonomic patterns $(20,23,24,27)$, phenotypic evolution in early neopterygians has only been discussed in qualitative terms. The implicit paleontological model of morphological conservatism among early teleosts contrasts with the observation that clades aligned with the teleost stem lineage include some of the most divergent early neopterygians in terms of both size and shape (Fig. 1) (see, for example, refs. 28 and 29). These discrepancies point to considerable ambiguity in initial patterns of phenotypic diversification that lead to a striking contrast in the vertebrate tree of life, and underpins one of the most successful radiations of backboned animals.

Here we tackle this uncertainty by quantifying rates of phenotypic evolution and capacity for evolutionary innovation for the first 160 million y of the crown neopterygian radiation. This late Permian (Wuchiapingian, ca. $260 \mathrm{Ma}$ ) to Cretaceous (Albian, ca. $100 \mathrm{Ma}$ ) sampling interval permits incorporation of diverse

\section{Significance}

The success of teleost fishes, which represent roughly half of all vertebrate species, has attracted attention since Darwin. Numerous scenarios invoke elevated diversification in teleosts facilitated by supposed key innovations, yet claims of teleost exceptionalism are profoundly biased by the evolutionary "snapshot" of living fishes. Analysis of $\mathbf{1 6 0}$ million y (PermianEarly (retaceous) of evolution in neopterygian fishes reveals that anatomical diversification in Mesozoic teleosts as a whole differed little from their "living fossil" holostean sister group. There is some evidence for evolutionary heterogeneity within teleosts, with early evolving lineages showing the greatest capacity for evolutionary innovation in body shape among Mesozoic neopterygians, whereas members of the modern teleost radiation show higher rates of shape evolution.

Author contributions: J.T.C. and M.F. designed research; J.T.C. performed research; G.T.L. contributed new reagents/analytic tools; J.T.C. and G.T.L. analyzed data; and J.T.C., G.T.L., and M.F. wrote the paper

The authors declare no conflict of interest.

This article is a PNAS Direct Submission.

Freely available online through the PNAS open access option.

${ }^{1}$ To whom correspondence should be addressed. Email: j.clarke.paleo@gmail.com.

${ }^{2}$ Present address: Museum of Paleontology and Department of Earth and Environmental Science, University of Michigan, Ann Arbor, Ml 48108-1079.

This article contains supporting information online at www.pnas.org/lookup/suppl/doi:10. 1073/pnas.1607237113/-/DCSupplemental. 


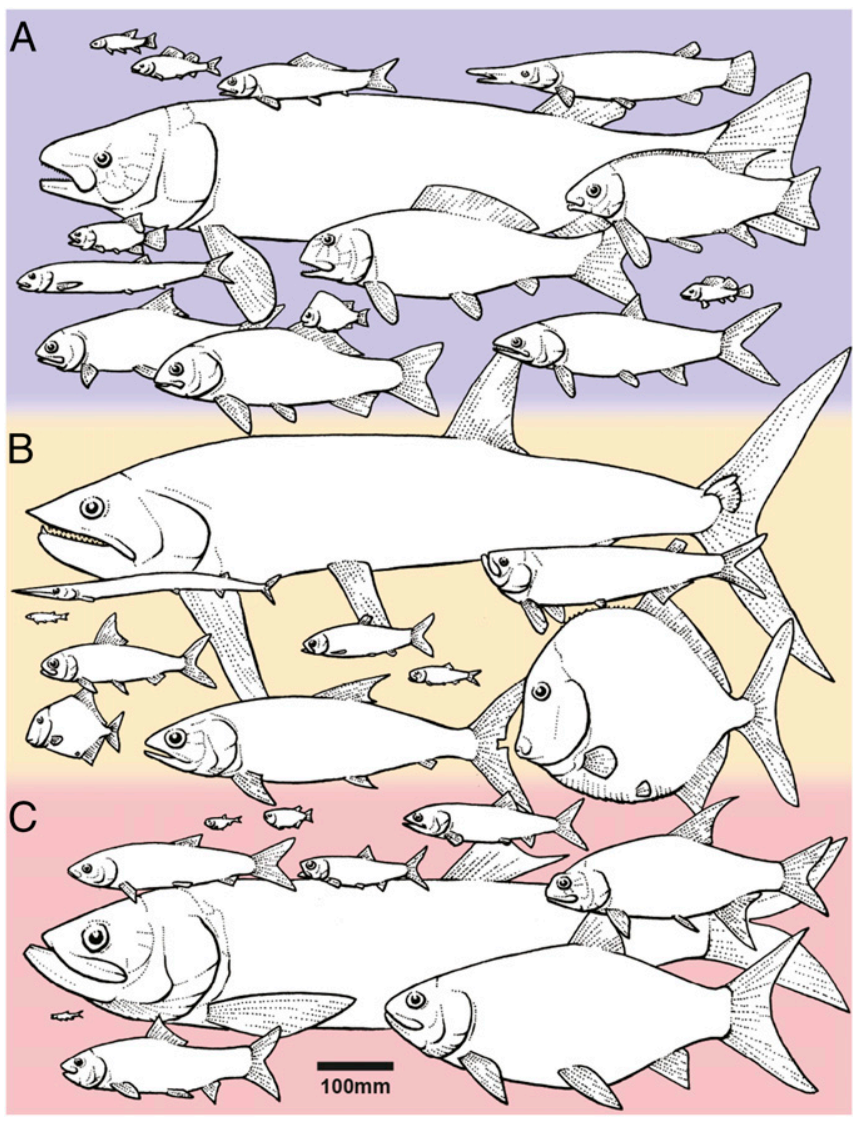

Fig. 1. Phenotypic variation in early crown neopterygians. $(A)$ Total-group holosteans. (B) Stem-group teleosts. (C) Crown-group teleosts. Taxa illustrated to scale.

fossil holosteans and stem teleosts alongside early diverging crown teleost taxa (Figs. 1 and $2 A$ and Figs. S1 and S2), resulting in a dataset of 483 nominal species-level lineages roughly divided between the holostean and teleost total groups (Fig. $2 B$ and Fig. S2). Although genera are widely used as the currency in paleobiological studies of fossil fishes (30; but see ref. 31), we sampled at the species level to circumvent problems associated with representing geological age and morphology for multiple congeneric lineages. We gathered size [both log-transformed standard length (SL) and centroid size (CS); results from both are highly comparable (Figs. S3 and S4); SL results are reported in the main text] and shape data (the first three morphospace axes arising from a geometric morphometric analysis) (Fig. $2 A$ and Figs. S1) from species where possible. To place these data within a phylogenetic context, we assembled a supertree based on published hypotheses of relationships. We assigned branch durations to a collection of trees under two scenarios for the timescale of neopterygian diversification based on molecular clock and paleontological estimates. Together, these scenarios bracket a range of plausible evolutionary timelines for this radiation (Fig. $2 B$ ). We used the samples of trees in conjunction with our morphological datasets to test for contrasts in rates of, and capacity for, phenotypic change between different partitions of the neopterygian Tree of Life (crown-, total-, and stem-group teleosts, total-group holosteans, and neopterygians minus crown-group teleosts), and the sensitivity of these conclusions to uncertainty in both relationships and evolutionary timescale. Critically, these include comparisons of phenotypic evolution in early crown-group teleosts-those species that are known with certainty to possess duplicate genomes-with rates in taxa characterized largely (neopterygians minus crown teleosts) or exclusively (holosteans) by unduplicated genomes. By restricting our scope to early diverging crown teleost lineages, we avoid potentially confounding signals from highly nested radiations that substantially postdate both genome duplication and the origin of crown teleosts $(26,32)$. This approach provides a test of widely held assumptions about the nature of morphological evolution in teleosts and their holostean sister lineage.

\section{Results and Discussion}

Mesozoic Teleosts Do Not Show Enhanced Phenotypic Diversification. Contrary to expectations ingrained in the neontological literature $(3,4,8,9,12,13,15)$, early teleosts do not possess significantly higher rates of size or shape evolution than holosteans (Fig. 3A). We found no significant difference between rates of body size (measured as SL; other measures deliver comparable results) (Figs. S3 and S4) evolution between early members of the holostean and teleost total groups across the majority of sampled topologies under either paleontological or molecular timescales (Fig. $3 A$ ). In terms of evolution in overall body shape (as indicated by scores on the first three shape axes of our morphospace), we found no consistent signal, whether rates were higher in total-group teleosts or holosteans (Fig. 3A). However, total-group holosteans possess significantly higher rates of overall shape change than total-group teleosts in a majority of topologies when these were timescaled to match published divergence-time estimates made using the molecular clock (Fig. $3 A$ ).

We calculated Blomberg's $K$ for specific clades to summarize how efficiently they explore phenotypic space (Methods). Because interpretation of $K$ in isolation can be misleading (33), it is useful to consider $K$ alongside information on evolutionary rate (34) (Table S1). We report $K$ values directly here and in subsequent sections, drawing on our comparisons of evolutionary rate to provide necessary context. Table S1 provides more detailed interpretations of $K$ using rate information. Concerning size innovation, $K$ distributions for the teleost and holostean total-groups overlap considerably on paleontological timescales (Fig. $3 A$ ), whereas holostean $K$ values are distinctly higher than teleost values on molecular timescales (Fig. $3 A$ ). Taken together with the suggestion of broadly comparable rates of evolution in the two clades (Fig. $3 A$ and Table S1), these results imply that total-group holosteans either match or exceed total-group teleosts in their size innovation. (Fig. $3 A$ and Table S1). $K$ distributions for shape in holosteans and teleosts are comparable regardless of timescale (Fig. $3 A$ and Table S1), suggesting they were similarly innovative.

The Broader Holostean Radiation Does Not Fulfill Numerous Living Fossil Expectations. Darwin articulated several concepts of what it means to be a living fossil (2), such as taxa that are "remnants of a once preponderant order," or are "slowly formed" (i.e., showing low rates of lineage diversification or trait evolution). Extant holosteans, which are among Darwin's archetypal living fossils (2), embody these features clearly: they represent the last survivors of a once diverse radiation and demonstrate low rates of lineage diversification $(26,32,35)$. The modern genera Amia, Lepisosteus, and Atractosteus show negligible anatomical change since their first appearance in the Late Cretaceous and Paleocene $(1,36)$, and living gars show low rates of body size evolution (17).

In contrast to this pattern from living species, Mesozoic holosteans show comparable rates of size change to total- (Fig. $3 A$ ), crown- (Fig. S5A), and stem-group teleosts (Fig. S5B). These patterns are seen across a majority of topologies regardless of timescale. Rates of shape evolution in holosteans are broadly comparable to those of teleosts, but often exceed those of totalgroup teleosts on molecular timescales (Fig. $3 A$ ) and stem teleosts on both timescales (Fig. S5B).

There is no clear difference in size innovation between holosteans and total- (Fig. $3 A$ ), crown- (Fig. S5 $A$ ), and stem- (Fig. S5B) group teleosts on paleontological timescales (Table S1). On molecular timescales, holostean-size $K$ values are marginally larger than those of total- (Fig. $3 A$ ) and crown-group teleosts (Fig. S5 $A$ ), and clearly larger than stem teleosts (Fig. S5B), suggesting holosteans are more innovative in these instances (Table S1). Unlike 


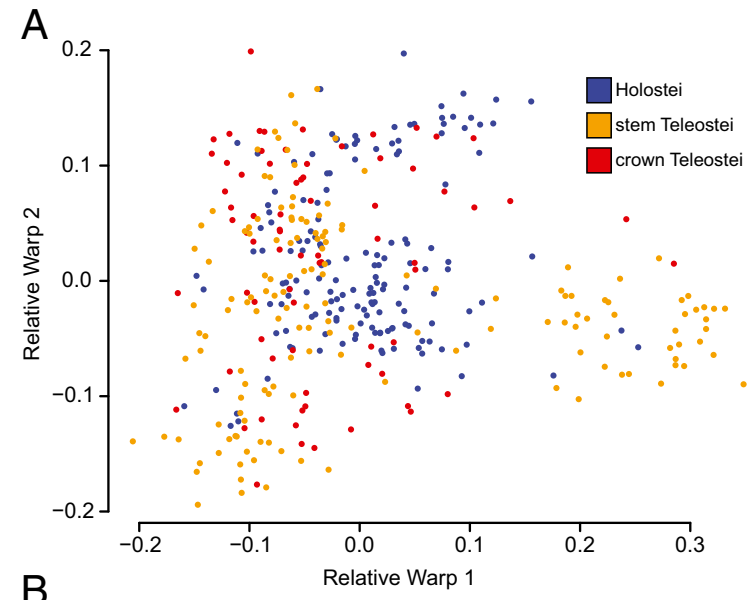

B
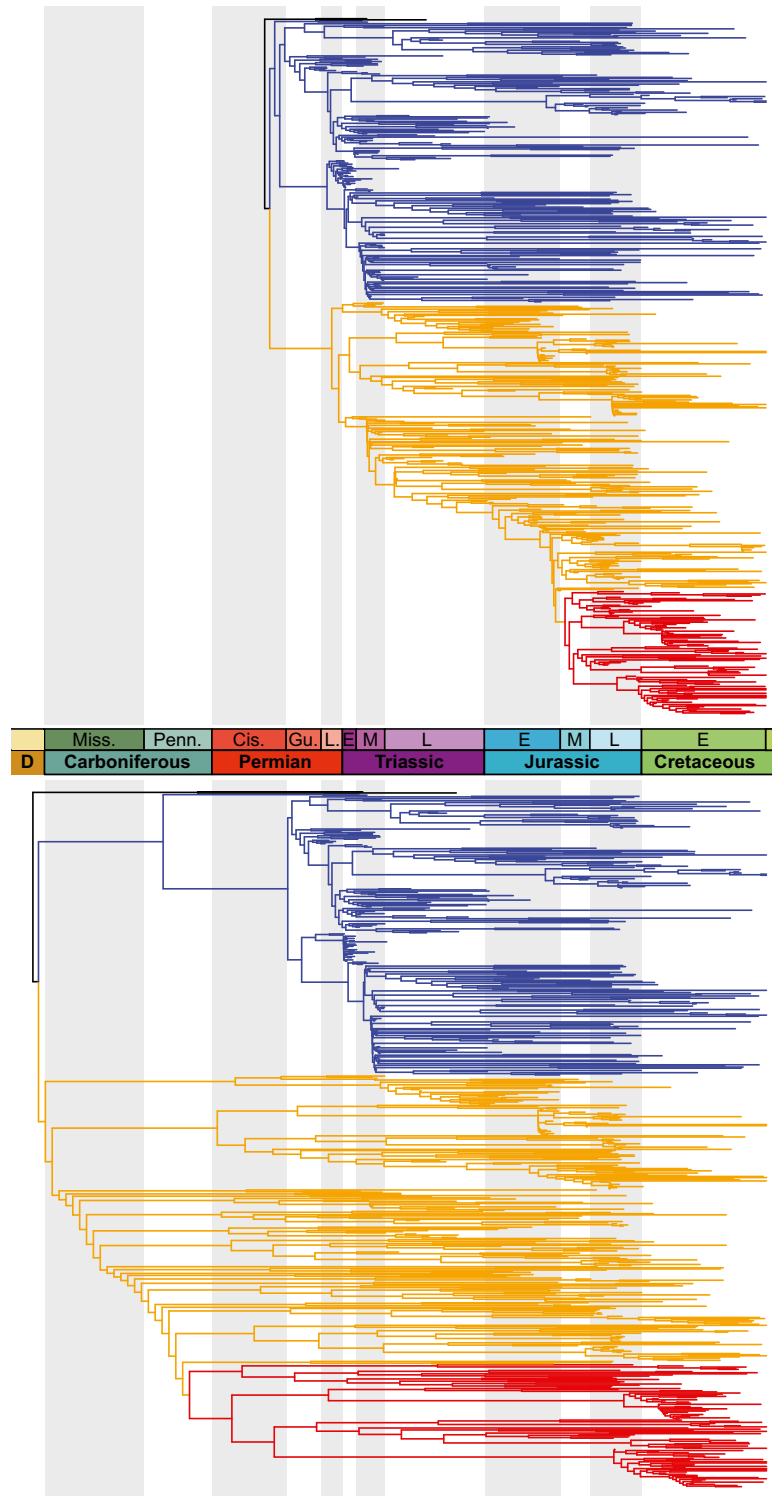

Fig. 2. (A) Morphospace of Permian-Early Cretaceous crown Neopterygii. $(B)$ One supertree subjected to our paleontological (Upper) and molecular (Lower) timescaling procedures to illustrate contrasts in the range of evolutionary timescales considered. Colors of points $(A)$ and branches $(B)$ indicate membership in major partitions of neopterygian phylogeny. Topologies are given in Datasets S4 and S5. See Dataset S6 for source trees. evolutionary rates and size innovation, there are some scenarios in which holosteans are marginally poorer shape innovators than teleosts. For example, on paleontological timescales, holosteans show marginally less shape innovation than crown teleosts (Fig. S5 $A$ and Table S1) and appear less innovative than stem teleosts (Fig. S5B and Table S1) regardless of timescale.

Crown Teleosts Display Comparable Patterns of Phenotypic Evolution to other Mesozoic Neopterygians. Despite possession of duplicate genomes, we find only ambiguous evidence for elevated shape evolution in early crown teleosts relative to rates in other neopterygian lineages. Rates are significantly higher for only a small majority of topologies on paleontological timescales (Fig. $3 B$ ), and fewer than half on molecular timescales (Fig. 3B). Evidence for higher rates in crown teleosts is even less compelling for size evolution, where a majority of trees display no significant difference in rate between crown teleosts and other neopterygians regardless of timescale (Fig. $3 B$ ).

Matching our inferences concerning evolutionary rate, we find no clear evidence to support the notion that early crown teleosts are better size or shape innovators than other neopterygian fishes as a whole. Regarding size, crown teleost $K$ values are comparable to those of other neopterygians regardless of timescale (Fig. $3 B$ ), suggesting they are similarly innovative (Table S1). Regarding shape, crown teleost $K$ values are either comparable to (on paleontological timescales) (Fig. $3 B$ ) or marginally lower than (on molecular timescales) (Fig. $3 B$ ) those of other neopterygians, suggesting they are similarly or less innovative, respectively (Table S1).

Capacity for Innovation and Rates of Phenotypic Change Vary Within the Teleost Total Group. Unremarkable evolutionary patterns across teleosts mask heterogeneities within the teleost total group. For example, crown-group teleosts show significantly elevated rates of shape evolution relative to stem teleosts in a small majority of topologies under both molecular and paleontological timescales (Fig. $3 C$ ). However, evidence for elevated rates of shape change in crowngroup teleosts is by no means unambiguous; we also find no rate difference between crown teleosts and stem teleosts, or significantly higher rates in stem teleosts, in a nontrivial fraction of topologies (Fig. 3C). Furthermore, finding higher shape rates in crown teleosts relative to stem taxa does not demonstrate uniquely enhanced shape diversification in crown teleosts, because holosteans also demonstrate higher shape rates than stem teleosts in a similar fraction of topologies (Fig. S5B). In contrast to these patterns for shape evolution, we find little evidence for elevated rates of size evolution in crown teleosts relative to members of the stem (Fig. 3C).

Possible contrasts in rates of shape evolution between crown teleosts and stem teleosts do not align with patterns of evolutionary innovation. $K$ distributions point to moderately (paleontological timescale) or substantially (molecular timescale) lower capacity for evolutionary innovation in members of the crown compared with those on the stem (Fig. 3C). Concerning differences in body-size evolution, there is little support for major differences between crown- and stem-group teleosts (Fig. 3C).

Tenuous Links Between Genome Duplication and Enhanced Evolutionary Rate and Innovation in Fishes. The staggering ecological and anatomical diversity of extant teleosts, especially in comparison with living nonteleost actinopterygian lineages, has long been taken as prima facie evidence of enhanced capacity for phenotypic evolution in this enormously successful vertebrate radiation $(3,4,8,9,12)$. Based on a more balanced taxon sample incorporating roughly equal numbers of early teleost and holostean species, we find that evidence for this widely held assumption is at best equivocal. Teleosts as a whole cannot be reliably distinguished from holosteans in terms of either rate of phenotypic change or capacity for evolutionary innovation. The most consistent contrasts we find concern patterns of shape change between crown- and stem-group teleosts, but these do not align: stem teleosts potentially show a higher capacity for evolutionary innovation, whereas crown teleosts are characterized by higher rates of phenotypic change. However, both 


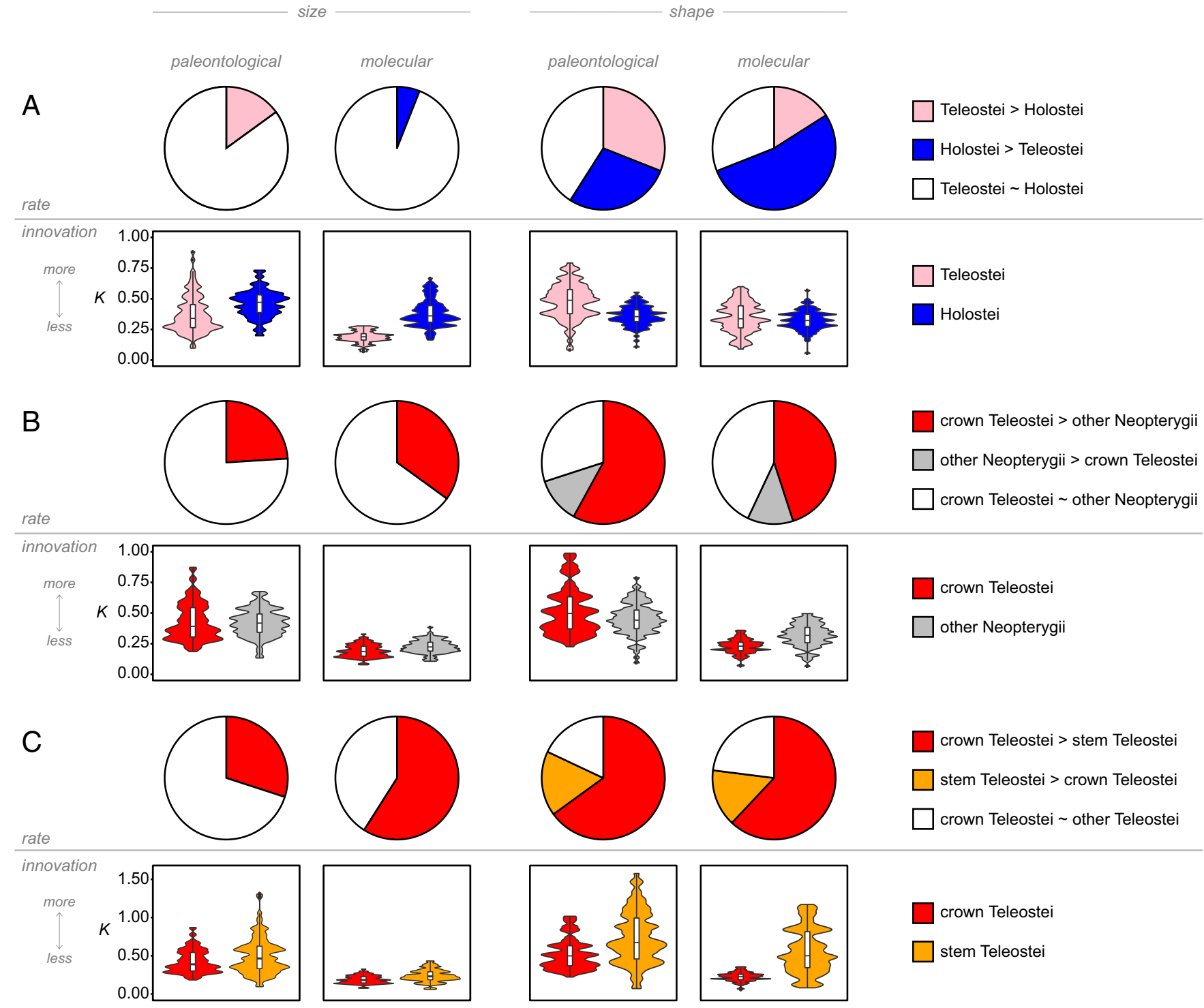

Fig. 3. Comparisons of phenotypic rate and innovation between $(A)$ holosteans and teleosts, $(B)$ crown teleosts and all other neopterygians, and $(C)$ crown teleosts and stem teleosts. Rate results are conveyed with pie charts, where the proportion of sampled supertrees in support of significantly higher rates (as determined from two-way $P$ values with $\alpha=0.05)$ in a given neopterygian partition are color coded to match that partition; white sections correspond to supertrees indicating no significant rate differences. Violin plots capturing the distribution of $K$ values obtained from 100 sampled supertrees allow for investigation of innovation. Higher $K$ values suggest greater phylogenetic signal, and correspondingly, greater innovation, in contrast to the iterative "rediscovery" of similar phenotypes (Methods). $K$ interpretations are contextualised with rate in Table S1.

must be viewed as ambiguous in light of the multiple pairwise comparisons made between partitions of neopterygian phylogeny.

With the obvious caveat that our paleontological sample excludes some of the most divergent modern teleost body plans, these results call into question the search for key innovations fueling the success of modern teleosts in sum. Many such features of teleost biology have been proposed (3-7), but the duplicate genomes that characterize all living members of this group represent the most popular candidate in recent literature $(8,11-13)$. The connection between genome duplication and shifts in evolutionary patterns in teleosts has, to date, been addressed in terms of rates of lineage diversification in modern species alone. As with our own examination of phenotypic evolution, these studies yield ambiguous results. Elevated rates of lineage diversification are not uniformly detected for crown teleosts as a whole, but there is a consistent and strong signal for exceptional shifts in rate associated with the hyperdiverse and phylogenetically nested otophysan and percomorph radiations $(26,32,35)$. The origin of these clades substantially postdates the teleost-specific whole-genome duplication, which molecular-clock dating of paralogue pairs (10) localizes to the middle of the teleost stem lineage rather than near the origin of the crown radiation (Table S2).

Further polyploid events within actinopterygians provide additional natural experiments for examining the consequences of genome duplications for subsequent patterns of evolutionary diversification. Here, too, results provide little direct support for increased rates of lineage diversification in polyploid groups relative to close relatives that have not undergone duplication events (37). In the case of groups like salmonids, geologically recent ecological shifts-rather than more ancient changes in genomic architecture-appear more closely linked with increased rates of lineage diversification $(38,39)$. How rates of phenotypic evolution might relate to these polyploid events has not been explored specifically, although at least two lineages characterized largely (Acipenseridae) or exclusively (Salmonidae) by polyploid species show elevated rates of body-size evolution relative to 
background actinopterygian rates (17). Thus, although genome duplication represents a seductive and widely enlisted hypothesis for explaining the taxonomic and especially morphological proliferation of clades, a synoptic view of the consequences of polyploid events on rates of lineage diversification and patterns of phenotypic change remains elusive.

Although our results do not strongly suggest any immediate consequences of genome duplication for morphological evolution in early crown-group teleosts, we anticipate that future developments could help to better constrain these patterns. Inferences about rate heterogeneity can vary substantially within our pool of sampled topologies. A more robustly constrained hypothesis of relationships and times of evolutionary divergence among early neopterygians, therefore, represents a first step to more decisive detection of shifts in the nature of morphological evolution across this major radiation, the early history of which has received substantially less systematic attention than more species-poor groups like birds (40-42) and mammals (43-45). Divergence estimates for paralogues (10) provide a loose constraint for the timing of the teleost-specific genome duplication (Table S2), but cannot identify which members of the teleost stem lineage were polyploid. There is the possibility that a paleogenomic approach using the size of osteocyte lacunae to estimate genome sizes in extinct lineages could more precisely pinpoint the phylogenetic position of the genome duplication. In addition to permitting more finely defined contrasts than those applied here, estimation of genome size in fossil teleosts would allow direct investigation of rates of genome reduction following duplication (46).

\section{Methods}

Phenotypic Datasets. Phenotypic data were collected from photographs of museum specimens of neopterygians ranging in age from Wuchiapingian (late Permian, $\sim 260 \mathrm{Ma}$ ) to Albian (Early Cretaceous, $100 \mathrm{Ma}$ ), supplemented by high-quality images in the primary literature. The phenotypic datasets represent a combined total of 1,170 unique specimen images assigned to 483 species.

Our phenotypic datasets are divided into those describing variation in size and those capturing differences in shape. Because of varying degrees of completeness between fossil specimens, these datasets do not contain identical sets of taxa, although the degree of overlap between any two datasets is high. We obtained SL for 949 specimens assigned to 468 species, and CS (based on our constellation of landmarks for geometric morphometic analyses; see below) for 626 individuals assigned to 382 species. Size values within species were averaged, and all resultant species sizes were log-transformed before analysis (SL in Dataset S1; CS in Dataset S2).

We used a 2D geometric morphometric approach using a constellation of 25 landmarks to quantify shape variation (Fig. S6) using the software package tpsDig2 (47). The shape dataset consisted of 774 specimen images assigned to 398 species (Fig. 2A and Figs. S1 and S2). Both fixed landmarks and semilandmarks were used to capture overall body shape and fin position, based on schemes applied previously to living (48) and fossil (31) fishes. Landmarked specimen data were aligned using orthogonal generalized Procrustes superimposition analysis (GPA), permitting shape values to be averaged within species. The averaged species data were then aligned with GPA and subject to a relative warp (RW) analysis in tpsRelw v1.54 (49). Of the four axes that described $>5 \%$ of overall variation, the first three (RW1 to 3) captured clear biological features (rather than differences potentially related to preservation) and formed the basis of all shape analyses in Dataset S3. RW1 to 3 explained $42.53 \%, 21.43 \%$, and $13.52 \%$ of the variation respectively. The Supporting Information details anatomical correlates of these axes (Table S3).

\section{Tree Construction.}

Summarizing existing topologies. Because there is no densely sampled phylogenetic hypothesis available for early fossil neopterygians, we adopted a "supertree" approach to produce a sample of trees for comparative analyses. Topologies were constructed using matrix representation with parsimony (MRP), drawing upon 120 source topologies (Dataset S6) to summarize relationships and capture phylogenetic uncertainty among 671 (mostly Mesozoic, but some living) neopterygian species. We adopted MRP because many trees lacked the data matrix used to create them (e.g., ref. 50) or were handconstructed (e.g., ref. 51). All junior synonyms present in the source trees were replaced by their correct senior synonyms to ensure all taxa were correctly represented in the source topologies. We included a "seed" (i.e., backbone) tree built with reliable taxonomic information that contained every species (52). This process permitted inclusion of large numbers of taxonomically assigned species that have not otherwise been included in a formal phylogenetic analysis. Use of taxonomic information is further vindicated given that paleontological trees derived from taxonomies can deliver comparable results using comparative methods to those derived from cladistic phylogenies (53). The taxonomy seed tree was also treated as a constraint on the supertree analysis to ensure that strongly corroborated placements could not be overruled by the source trees (e.g., holostean monophyly, which is well-supported by modern molecular and morphological analyses, but not recovered by older studies). We purposely left the seed tree poorly resolved to allow source trees to dictate relationships where there is genuine uncertainty. A second constraint was applied to ensure that the relationships between major living teleost clades matched those arising from recent molecular phylogenetic studies (54). To implement both constraints, the nodes (expressed as characters in the MRP matrix) defining the relationships of the taxonomic and molecular trees were upweighted to 1,000 (the maximum) in our MRP data matrix.

Safe taxonomic reduction (55) was performed upon the MRP matrix using Claddis v0.1 (56) before phylogenetic analysis in TNT v1.1 (57). Twenty replicates of new technology searches were performed, saving 1,000 trees each time, with each replicate starting from a random tree. Ten-thousand MPTs were then obtained from these saved replicates, followed by a final search for remaining MPTs with tree bisection and reconnection, delivering a total of 10,500 MPTs. Taxa removed by safe taxonomic reduction were reinserted into every MPT, either into their sole possible position or, if multiple positions were equally likely, one was chosen at random. One-hundred trees were then selected at random from this pool for downstream comparative analyses, with any remaining polytomies randomly resolved using the "multi2di" function in APE (58).

Timescaling topologies. Living species were pruned from our 100 supertrees before timescaling using the timePaleoPhy function of the paleotree package in $\mathrm{R}$ (59). As illustrated in Fig. $2 B$, we adopted two end-member timescaling procedures: ( $i$ ) a paleontological timescale to reflect divergence times based solely upon fossils, and (ii) a molecular timescale to reflect some of the oldest neopterygian divergence estimates in recent clock studies.

The tip age of every species was randomized (with a uniform distribution) between its oldest potential age (i.e., the oldest lower boundary age of all of the deposits where the species is found) and its oldest reliable minimum age (i.e., the oldest upper boundary age of all of the deposits where the species is found). This randomization procedure was carried out for each tree individually. We used the node-dating procedure of Hedman (60) to provide an estimate for the neopterygian crown node (i.e., the root of the supertree) as the first step in timescaling topologies under our paleontological approach. This approach delivered a mean estimate of $280 \mathrm{Ma}$ for the neopterygian crown, which we set as the root age. For our molecular timescaling procedure, we constrained the age of three nodes based upon the clock estimates of Near et al. (54) (crown Neopterygii: 361.2 Ma; crown Holostei: $271.9 \mathrm{Ma}$; crown Teleostei: $307.1 \mathrm{Ma}$ ). For both paleontolgical and molecular timescaling, we used the "equal" method implemented in timePaleoPhy.

Quantifying Phenotypic Rates. Size rates were quantified using the Bayesian approach of Eastman et al. (61) implemented over 1,000,000 generations, discarding the first 250,000 generations as burn-in. Randomization tests (implemented via the "compare.rates" function of the auteur package in $\mathrm{R}$ v2.15.3) provided a two-way $P$ value $(\alpha=0.05)$ to test for differences between neopterygian partitions. The Adams (62) method permits estimation of evolutionary rate on multivariate data, and was applied to our shape dataset. Simulation of each supertree topology under a null model of equal rates was used to generate a null distribution of rate ratios for each of our five comparisons. The observed rate ratio for a given comparison can then be compared with the simulated distribution of rate ratios to derive a twoway $P$ value to test for differences between two sets of taxa $(\alpha=0.05)$.

Quantifying Phenotypic Innovation. Blomberg's $K$ quantifies whether closely related taxa in a clade of interest are either more $(K>1)$ or less similar $(K<$ 1) with respect to a trait value than expected under a Brownian motion model of evolution $(K=1)$. Therefore, a more innovative clade (i.e., one that is efficient at exploring new regions of trait space) should have a larger $K$ value than a less innovative clade. This is because the lineages of an innovative clade should spread apart from one another, occupying different regions of trait space so that more closely related taxa appear more similar in trait value than more distantly related taxa. A less-innovative clade should express lower $K$ values, as multiple lineages overlap and re-explore similar phenotypes, eroding phylogenetic signal. Variation in rate of phenotypic change between focal groups can, however, distort this simple relationship. For example, in a clade that shows high rates of phenotypic change relative to its boundaries in 
phenotypic space, $K$ can be degraded $(33,34) . K$ values are interpreted to reflect innovation in the main text with appropriate caveats given potential differences in rate, with all comparisons further contextualized in Table S1.

ACKNOWLEDGMENTS. We thank L. Sallan, R. Benson, R. Close, and L. Soul for useful discussion; and the collections managers, curators, and research

1. Grande L (2010) An empirical synthetic pattern study of gars (Lepisosteiformes) and closely related species, based mostly on skeletal anatomy. The resurrection of Holostei. (American Society of Ichthyologists and Herpetologists, Copeia, Lawrence, KS) 10(2A).

2. Darwin C (1859) On the Origin of Species by Means of Natural Selection, or the Preservation of Favoured Races in the Struggle for Life (John Murray, London), 1st Ed

3. Pough FH, Heiser JB, McFarland WN (1996) Vertebrate Life, 4th ed. (Prentice Hall, Upper Saddle River, NJ).

4. Colbert EH (1969) Evolution of Vertebrates (Wiley, New York), 2nd Ed.

5. Motta PJ (1984) Mechanics and functions of jaw protrusion in teleost fishes-A re view. Copeia 1984(1):1-18.

6. Collazo A, Bolker JA, Keller R (1994) A phylogenetic perspective on teleost gastrulation. Am Nat 144(1):133-152.

7. Collazo A (1996) Evolutionary correlations between early development and life history in plethodontid salamanders and teleost fishes. Am Zool 36(2):116-131.

8. Hoegg S, Brinkmann H, Taylor JS, Meyer A (2004) Phylogenetic timing of the fish-specific genome duplication correlates with the diversification of teleost fish. J Mol Evol 59(2):190-203.

9. Crow KD, Stadler PF, Lynch VJ, Amemiya C, Wagner GP (2006) The "fish-specific" Hox cluster duplication is coincident with the origin of teleosts. Mol Biol Evol 23(1):121-136.

10. Hurley IA, et al. (2007) A new time-scale for ray-finned fish evolution. Proc Biol Sc 274(1609):489-498.

11. Wittbrodt J, Meyer A, Schartl M (1998) More genes in fish? BioEssays 20(6):511-515

12. Meyer A, Van de Peer $Y$ (2005) From $2 R$ to 3R: Evidence for a fish-specific genome duplication (FSGD). BioEssays 27(9):937-945.

13. Glasauer SMK, Neuhauss SCF (2014) Whole-genome duplication in teleost fishes and its evolutionary consequences. Mol Genet Genomics 289(6):1045-1060.

14. Freeling $M$, Thomas BC (2006) Gene-balanced duplications, like tetraploidy, provide predictable drive to increase morphological complexity. Genome Res 16(7):805-814.

15. Van de Peer Y, Maere S, Meyer A (2009) The evolutionary significance of ancient genome duplications. Nat Rev Genet 10(10):725-732.

16. Postlethwait J, Amores A, Cresko W, Singer A, Yan YL (2004) Subfunction partitioning, the teleost radiation and the annotation of the human genome. Trends Genet 20(10):481-490.

17. Rabosky DL, et al. (2013) Rates of speciation and morphological evolution are correlated across the largest vertebrate radiation. Nat Commun 4:1958.

18. Kner R (1866) Betrachtungen über die Ganoiden, als Natürliche Ordnung (Sitzungsberichte der Kaiserlichen Akademie der Wissenschaften, Wien), pp 519-536.

19. Woodward AS (1901) Catalogue of the Fossil Fishes in the British Museum (Natural History). Part IV. Trustees of the British Museum (Natural History, London).

20. Thomson KS (1977) The pattern of diversification among fishes. Developments in Palaeontology and Stratigraphy, ed Hallam A (Elsevier, Amsterdam), pp 377-404.

21. Romer AS (1966) Vertebrate Paleontology (Univ of Chicago Press, Chicago), 3rd Ed.

22. Arratia G (1997) Basal teleosts and teleostean phylogeny. Palaeo Ichthyologica 7:5-168

23. Guinot G, Cavin L (2015) 'Fish' (Actinopterygii and Elasmobranchii) diversification patterns through deep time. Biol Rev Camb Philos Soc 25:2314-2318.

24. Romano C, et al. (2016) Permian-Triassic Osteichthyes (bony fishes): Diversity dynamics and body size evolution. Biol Rev Camb Philos Soc 91(1):106-147.

25. Patterson C (1977) The contributions of paleotnology to teleostean phylogeny. Major Patterns in Vertebrate Evolution, eds Hecht MK, Goody PC, Hecht BM (Plenum, New York), pp 579-643.

26. Santini $F$, Harmon $\sqcup$, Carnevale G, Alfaro ME (2009) Did genome duplication drive the origin of teleosts? A comparative study of diversification in ray-finned fishes. BMC Evol Biol 9:194.

27. McCune AR, Schaeffer B (1986) Triassic and Jurassic fishes patterns of diversity. The Beginning of the Age of Dinosaurs: Faunal Change across the Triassic-Jurassic Boundary; Symposium Held in Conjunction with the 44th Annual Meeting of the Society of Vertebrate Paleontology, Berkeley, California, USA, October 31, 1984, ed Padian K (Cambridge Univ Press, Cambridge, UK), pp 171-182.

28. Friedman $\mathrm{M}$, et al. (2010) 100-million-year dynasty of giant planktivorous bony fishes in the Mesozoic seas. Science 327(5968):990-993.

29. Poyato-Ariza FJ, Wenz S (2002) A new insight into pycnodontiform fishes. Geodiversitas 24(1):139-248.

30. Forey PL, Fortey RA, Kenrick P, Smith AB (2004) Taxonomy and fossils: A critical appraisal. Philos Trans R Soc Lond B Biol Sci 359(1444):639-653.

31. Friedman $M$ (2010) Explosive morphological diversification of spiny-finned teleost fishes in the aftermath of the end-Cretaceous extinction. Proc Bio/ Sci 277(1688):1675-1683.

32. Alfaro ME, et al. (2009) Nine exceptional radiations plus high turnover explain species diversity in jawed vertebrates. Proc Natl Acad Sci USA 106(32):13410-13414.

33. Revell LJ, Harmon LJ, Collar DC (2008) Phylogenetic signal, evolutionary process, and rate. Syst Biol 57(4):591-601.

34. Hopkins MJ, Smith AB (2015) Dynamic evolutionary change in post-Paleozoic echinoids and the importance of scale when interpreting changes in rates of evolution. Proc Natl Acad Sci USA 112(12):3758-3763.

35. Near TJ, et al. (2014) Boom and bust: Ancient and recent diversification in bichirs (Polypteridae: Actinopterygii), a relictual lineage of ray-finned fishes. Evolution 68(4):1014-1026. scientists at numerous institutions for their assistance and access to fossil specimens. This work was supported by a Palaeontological Association Whittington Award and a Natural Environment Research Council Cohort grant (to J.T.C.); Australian Research Council Grant DE140101879 (to G.T.L.); Philip Leverhulme Prize PLP 2012-130 (to M.F.); Natural Environment Research Council Award NE/I005536/1 (to M.F.); and the John Fell Fund (M.F.)

36. Grande L, Bemis WE (1998) A comprehensive phylogenetic study of Amiid fishes (Amiidae) based on comparative skeletal anatomy. An empirical search for interconnected patterns of natural history. Society of Vertebrate Paleontology Memoir, 4:1-690.

37. Zhan SH, Glick L, Tsigenopoulos CS, Otto SP, Mayrose I (2014) Comparative analysis reveals that polyploidy does not decelerate diversification in fish J Evol Bio/ 27(2):391-403.

38. Alexandrou MA, Swartz BA, Matzke NJ, Oakley TH (2013) Genome duplication and multiple evolutionary origins of complex migratory behavior in Salmonidae. $\mathrm{Mol}$ Phylogenet Evol 69(3):514-523.

39. Macqueen DJ, Johnston IA (2014) A well-constrained estimate for the timing of the salmonid whole genome duplication reveals major decoupling from species diversification. Proc Biol Sci 281(1778):20132881.

40. Fountaine TMR, Benton MJ, Dyke GJ, Nudds RL (2005) The quality of the fossil record of Mesozoic birds. Proc Biol Sci 272(1560):289-294.

41. O'Connor JK, Zhou Z (2013) A redescription of Chaoyangia beishanensis (Aves) and comprehensive phylogeny of Mesozoic birds. J Syst Palaeontology 11(7):889-906.

42. Lee MSY, Worthy TH (2012) Likelihood reinstates Archaeopteryx as a primitive bird. Biol Lett 8(2):299-303.

43. Luo Z-X (2007) Transformation and diversification in early mammal evolution. Nature 450(7172):1011-1019.

44. Zheng X, Bi S, Wang X, Meng J (2013) A new arboreal haramiyid shows the diversity of crown mammals in the Jurassic period. Nature 500(7461):199-202.

45. Luo Z-X, Gatesy SM, Jenkins FA, Jr, Amaral WW, Shubin NH (2015) Mandibular and dental characteristics of Late Triassic mammaliaform Haramiyavia and their ramifications for basal mammal evolution. Proc Natl Acad Sci USA 112(51):E7101-E7109.

46. Inoue J, Sato $Y$, Sinclair $R$, Tsukamoto $K$, Nishida $M$ (2015) Rapid genome reshaping by multiple-gene loss after whole-genome duplication in teleost fish suggested by mathematical modeling. Proc Natl Acad Sci USA 112(48):14918-14923.

47. Rohlf FJ (2013) TPSdig2, v.2.17 (Department of Ecology and Evolution, SUNY at Stony Brook, Stony Brook, NY)

48. Kerschbaumer M, Sturmbauer C (2011) The utility of geometric morphometrics to elucidate pathways of cichlid fish evolution. Int J Evol Biol 2011:290245.

49. Rohlf FJ (2014) tpsRelw, v1.54 (Department of Ecology and Evolution, SUNY at Stony Brook, Stony Brook, NY)

50. Lambers PH (1995) The monophyly of the Caturidae (Pisces, Actinopterygii) and the phylogeny of the Halecomorphi. Geobios 28:201-203.

51. Taverne L (2011) Osteology and phylogenetic relationships of Steurbautichthys ("Pholidophophorus") aequatorialis gen. nov (Teleostei, "Pholidophoriformes") from the Middle Jurassic of Kisangani, Democratic Republic of Congo. Osteologie et relations phylogenetiques de Steurbautichthys ("Pholidophophorus") aequatorialis gen. nov. (Teleostei, "Pholidophoriformes") du Jurassique moyen de Kisangani, en Republique Democratique du Congo. Bulletin de I'Institut Royal des Sciences Naturelles de Belgique Sciences de la Terre 81:129-173.

52. Bininda-Emonds ORP, Sanderson MJ (2001) Assessment of the accuracy of matrix rep resentation with parsimony analysis supertree construction. Syst Biol 50(4):565-579.

53. Soul LC, Friedman M (2015) Taxonomy and phylogeny can yield comparable results in comparative paleontological analyses. Syst Biol 64(4):608-620

54. Near TJ, et al. (2012) Resolution of ray-finned fish phylogeny and timing of diversification. Proc Natl Acad Sci USA 109(34):13698-13703.

55. Wilkinson $M$ (1995) Coping with abundant missing entries in phylogenetic inference using parsimony. Syst Biol 44(4):501-514.

56. Lloyd GT (2016) Estimating morphological diversity and tempo with discrete character-taxon matrices: Implementation, challenges, progress, and future directions. Biol Linn Soc Lond 118:131-151.

57. Goloboff PA, Farris JS, Nixon KC (2008) TNT, a free program for phylogenetic analysis. Cladistics 24(5):774-786.

58. Paradis E, Claude J, Strimmer K (2004) APE: Analyses of phylogenetics and evolution in $\mathrm{R}$ language. Bioinformatics 20(2):289-290.

59. Bapst DW (2012) paleotree: An R package for paleontological and phylogenetic analyses of evolution. Methods Ecol Evol 3(5):803-807.

60. Hedman MM (2010) Constraints on clade ages from fossil outgroups. Paleobiology 36(1):16-31.

61. Eastman JM, Alfaro ME, Joyce P, Hipp AL, Harmon $\sqcup$ (2011) A novel comparative method for identifying shifts in the rate of character evolution on trees. Evolution 65(12):3578-3589.

62. Adams DC (2014) Quantifying and comparing phylogenetic evolutionary rates for shape and other high-dimensional phenotypic data. Syst Biol 63(2):166-177.

63. Bookstein FL (1986) Size and shape spaces for landmark data in two dimensions. Stat Sci 1:238-242.

64. Edgecombe GD, Legg DA (2014) Origins and early evoloution of arthropods Palaeontology 57(3):457-468.

65. Clarke JT, Warnock RCM, Donoghue PCJ (2011) Establishing a time-scale for plant evolution. New Phytol 192(1):266-301.

66. Dornburg A, Townsend JP, Friedman M, Near TJ (2014) Phylogenetic informativeness reconciles ray-finned fish molecular divergence times. BMC Evol Biol 14:169.

67. Bapst DW (2013) A stochastic rate-calibrated method for time-scaling phylogenies of ossil taxa. Methods Ecol Evol 4(8):724-733. 